\title{
Novel Geometry Reaching High Efficiency for Multiple Injector Concepts
}

\author{
Author, co-author (Do NOT enter this information. It will be pulled from participant tab in \\ MyTechZone) \\ Affiliation (Do NOT enter this information. It will be pulled from participant tab in MyTechZone)
}

\begin{abstract}
Heat losses are known to decrease the efficiency of CI engines largely. Here, multiple injectors have been suggested to shrink these losses through reduction of spray wall impingement. Studies on multiple injectors have proven the concept's heat transfer reduction but also highlighted the difficulty of using a standard piston bowl. This study proposes a two-injector concept combined with a flat bowl to reduce heat losses further. To change the spray pattern, the two injectors are injecting in a swirling motion while placed at the rim of the bowl. Four injection timings have been investigated using Reynolds-Averaged Navier-Stokes simulations. This computational method quantified the amount of heat loss reduction possible. A conventional single injector concept is compared to two injector concepts with a standard and flat bowl. A Double Compression Expansion Engine (DCEE) concept, based on a modified Volvo D13 single-cylinder engine, was the base for all simulations. The DCEE can re-use the residual exhaust energy for a second expansion meaning increased importance of reduced heat losses. Heat release effects were discarded in the evaluation as an explanation for the reduced heat losses in order to isolate the effects of the changed spray pattern. Results showed a decrease in heat losses by $25.1 \%$ or $4.2 \%$ of the fuel energy as well as an increased IMEP of $4.5 \%$ or $1.9 \%$ of the fuel energy. Together with the increased exhaust energy, results showed a possible total engine efficiency increase of $2.6 \%$ using the DCEE concept. This work successfully proves the benefits of using two injectors with a flat bowl over a standard bowl and the conventional one-injector strategy.
\end{abstract}

\section{Introduction}

Research of modern CI engines focuses on reducing the $\mathrm{CO} 2$ emissions by increasing the efficiency. New regulations restrict the carbon footprint further [1], meaning an increased strive for $\mathrm{CO} 2$ emissions reduction. The $\mathrm{CO} 2$ emissions are set to be reduced by $15 \%$ until 2025 and $30 \%$ in 2030. Particularly, the heavy-duty vehicles are targeted as the main contributor to $\mathrm{CO} 2$-emissions among vehicles [2]. A strive for increased energy efficiency follows.

Some engine alternatives have been suggested for increasing the efficiency of the IC engine. In later years there has been a trend for low-temperature combustion concepts including the Homogenous Charge Compression Ignition (HCCI) [3] and Partially Premixed Combustion (PPC) [4]. The idea of these concepts is to reduce the heat losses through a lower burned gas temperature.
The HCCI concept combines the benefits of a homogeneous charge with a compression ignition concept. Studies have suggested that increased efficiency follows from low heat losses [3] proving the benefits of this concept. Other studies [5] have presented some drawbacks of the HCCI, including ignition difficulties at low load and low combustion efficiency.

The PPC concept tries to solve the flaws of the HCCI through a later injection inducing a less homogenous charge. In [6], PPC combustion at different load conditions was investigated. It was concluded that an indicated efficiency of $57 \%$ could be achieved. Low heat transfer, as well as low exhaust losses, were claimed. However, it was difficult to reach a high load with the PPC concept. This was caused by the too short ignition delays at higher pressure.

Another promising concept for solving the problems of the lowtemperature theory as well as keeping a high efficiency at all loads is the Double Compression Expansion Engine (DCEE) [7]. By dividing the cycle into two cylinders, this concept is capable of performing a second expansion using the spare exhaust energy. It follows that high exhaust energy is beneficial for this concept as a contrary to typical IC engine concepts.

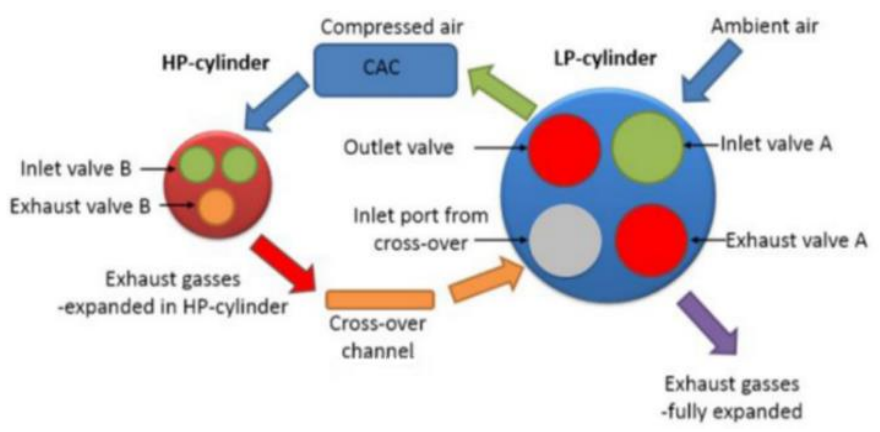

Figure 1, The DCEE concept outlined with the high-pressure (HP) and lowpressure (LP) cylinders [8]

Dividing the cycle into two cylinders also means that very high pressure can be used without the usual high friction losses. The lowpressure (LP) cylinder will compress the intake air before it is transferred to the high-pressure (HP) cylinder. Here, a second compression takes place leading to pressures as high as 300 bar. This compression is followed by a typical CI fuel injection and combustion. The first expansion takes place here in the HP-cylinder followed by a gas transfer to the LP-cylinder where a second expansion takes place. 
Using the DCEE concept, Lam et al. demonstrated brake efficiencies of $56 \%$ [8] as well as $52.7 \%$ [9]. Further efficiency improvements have then been achieved by Shankar et al. [10] with the use of insulation to reduce heat losses. This study proves the importance of heat transfer reductions for this concept. The second expansion uses the extra exhaust energy. Thus, it becomes further significant to keep the heat losses low to increase exhaust energy.

A typical approach of achieving lower heat losses in a CI engine is to reduce wall impingement, i.e., to keep the high-temperature zones away from the cylinder walls. This lower flame/wall interaction can be achieved by increasing the injection time as well as reducing the injection pressure. However, there are some limitations to these ideas when going towards high load since more fuel needs to be injected. Another solution was proposed by Uchida et al. [11] using multiple injectors. Further evaluation has been done on this concept [12] showing a heat transfer reduction of $15 \%$ or $2 \%$ of the fuel energy through a changed flow pattern. However, this study highlighted the issues of an omega-shaped bowl when using two injectors. The central pip increases impingement and narrows the possibilities of directing the sprays. Notable is that this study also emphasized that the multiple-injector is not to be restricted to the DCEE concept but can be used in any engine with exhaust regeneration (e.g., a turbo).

To solve the issues of an omega-shaped bowl, a flat bowl where the central pip is removed is here proposed for the two-injector concept. This is expected to reduce the heat transfer further through the changed flow pattern as well as the smaller surface area. Since multiple injectors increase the cost of an engine, it is of interest to investigate the quantity of efficiency gain. In this computational study, the heat transfer is examined and compared with previous cases showing a substantial reduction through the changed flow pattern.

\section{Methodology}

Three-dimensional RANS CFD simulations have been completed using the software Converge (version 2.4) during the compression stroke and expansion stroke of the high-pressure unit. No air exchange and so, no pumping losses have been taken into account in this study. More details about the methodology were reported in a previous study [12].

\section{Test Conditions}

Only the combustion chamber has been chosen as the computational domain in this study, i.e., valves, intake and exhaust systems have not been considered. To compensate this, the chamber has been assumed to contain a specified composition of gases at the start of compression. The residual exhaust gases from the previous cycle in this composition have been considered to hold only water, carbon dioxide (CO2), nitrogen (N2) and oxygen (O2) since these are usually the main components in diesel engine exhaust. Since intake flow is not considered in this setup, an initial turbulence level was set.

The geometry studied here is a standard 4-stroke Volvo D13 engine but with an altered compression ratio (see Table 1). An engine speed of $1200 \mathrm{rpm}$ was set for all different cases. All cases were simulated using Diesel surrogate as fuel, with different injection timings. Only the fluid domain was simulated, and wall temperatures were assumed to be constant.

Page 2 of 9
Table 1, Engine Parameters

\begin{tabular}{|l|l|}
\hline Engine parameters & \\
\hline Cylinder volume & 2.331 \\
\hline Stroke & $158 \mathrm{~mm}$ \\
\hline Bore & $131 \mathrm{~mm}$ \\
\hline Connecting Rod & $267.5 \mathrm{~mm}$ \\
\hline Compression Ratio & $11.5: 1$ \\
\hline Engine Speed & $1200 \mathrm{rpm}$ \\
\hline Intake Temperature & $464.15 \mathrm{~K}$ \\
\hline Nozzle hole diameter & $240 \mu \mathrm{m}$ \\
\hline Number of injector holes & 6 \\
\hline Standard umbrella angle & 145 degrees \\
\hline Lambda & 3.2 \\
\hline Intake Pressure & 5 bar \\
\hline
\end{tabular}

\section{CFD Models and Validation}

$\mathrm{N}$-heptane is here used as a surrogate for the multicomponent Diesel. The liquid phase spray is Diesel meaning only evaporated parcels are $\mathrm{n}$-heptane. However, the lower heating value of diesel is used to account for the fuel energy. The physical properties of n-heptane are incorporated in the CFD code. Spray breakup model, used to capture the spray injection, was based on a Kevin-Helmholtz Rayleigh-Taylor (KH-RT) approach [13]. All droplets are assumed to disperse and merge during the breakup process. The spherical shape is assumed for all droplets, and the Frossling droplet evaporation correlation [14] was used to calculate new droplet diameter during evaporation. The Rosslin-Rammler cumulative probability distribution was implemented to account for the size distribution of droplets in the domain.

In this study, a renormalized group k-epsilon RANS turbulence model was used to account for in-cylinder turbulence. Other physical submodels follow the standard built-in capabilities in CONVERGE v2.4 [15].

A base grid size of $2 \mathrm{~mm}$ was used in all three directions for the mesh. Fixed embeddings for the injectors were added to account for the nearnozzle flow. The flow inside the nozzle is not considered in the study. Level 3 adaptive mesh refinement (AMR) was utilized based on velocity and temperature gradients inside the combustion domain. The resulting minimum cell size in all three directions after AMR implementation is calculated based on the standard formulation

Min.cell size $=\frac{\text { Base grid }}{2^{n}}$

where $\mathrm{n}$ is the level of refinement provided. The meshing strategy is further described in a previous CFD study [16].

All heat transfer calculations in this paper are using the O'Rourke heat transfer model [17] based on the law of the wall where a constant wall temperature of $500 \mathrm{~K}$ is assumed. Since the models are validated against pressure and heat release, the heat transfer is also assumed validated. Only minor differences in heat losses were found with the use of radiation models. Thus radiation modeling was not included in this study. 
The chemical models are an essential part of any engine combustion simulation in order to properly predict the ignition delay as well as combustion timing. A SAGE multi-zone combustion model [18] was used by mapping the grid cells to temperature bins of $5 \mathrm{~K}$ and equivalence ratio bins of 0.05 increments.

A skeletal n-heptane mechanism [19] with 110 species was used in this study. It has been demonstrated that this mechanism is relevant at a number of operating conditions, without further adjustments [19].

Since many simplifications and assumptions are needed for RANS CFD simulations, a validation process has been performed based on the setup discussed above. Simulation results have been compared to experiments conducted at the combustion engines group at Lund University.

At first, the compression ratio was adjusted to fit the motoring trace, motivated by that the connecting rod flex is not considered in the models. The effective compression ratio used was set to 11.41 instead of 11.5 which is in line with previous studies on connecting rod flex making the effective compression ratio lower [20].

Since no intake stroke is performed in the simulations, leftover exhaust gases were introduced in the chamber. These were assumed only to contain $\mathrm{H}_{2} \mathrm{O}, \mathrm{CO}_{2}, \mathrm{~N}_{2}$, and $\mathrm{O}_{2}$, since these are the major components. Residual gases are expected to be present from the previous cycle due to gas exchange inefficiencies. This affects the fluid specific heat ratio, which was decided through comparison with the experimental data before the start of combustion.

Considering the effective SOI and injection duration, the injection timing was changed to -0.2 CAD ATDC instead of -1 CAD ATDC. Similarly, the injection duration was changed from 7.2 CAD to 8.5 CAD. Ignition delay was optimized to fit experimental results.

After several adjustments on the injection timing and duration, the simulation was validated by comparison to experimental data as demonstrated in Figure 2. Furthermore, the Rate of Heat Release (RoHR) plot shows that the simulation is well-predicting the peak value.

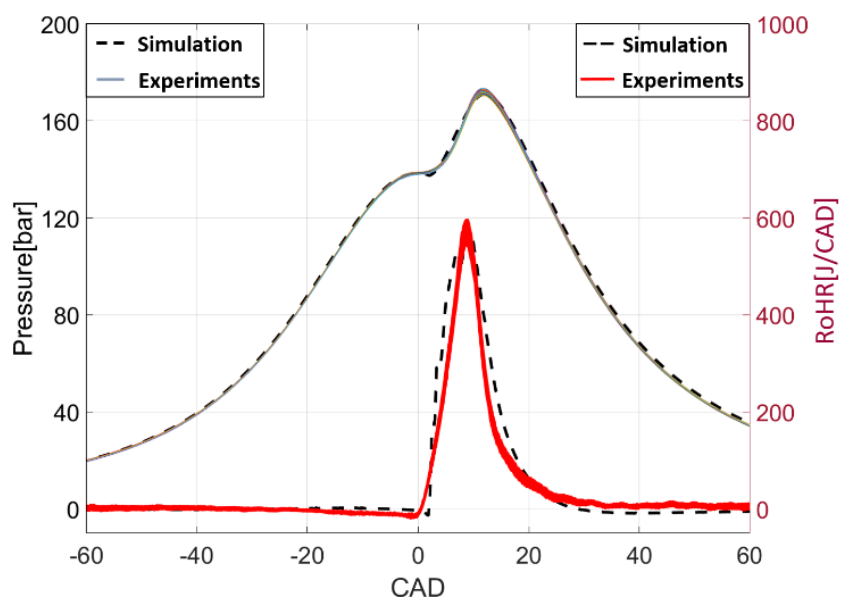

Figure 2, Pressure [bar] and RoHR [J/CAD] traces of the simulation case vs. experimental results
Pressure, RoHR and ignition delay matched experimental data well. This simulation case will be considered in the following analysis as the reference case. Heat transfer models were also assumed validated based on the Pressure and RoHR traces.

\section{Project Approach}

This paper focuses on a flat bowl geometry to reduce the heat transfer for multiple-injector usage. Two new geometries are proposed and evaluated against a reference geometry as well as an earlier multiple injector study [12]. This means that three different geometries were tested, geometry 2 and 3 using a flat bowl for two outer injectors and geometry 1 using a standard bowl (see Figure 3 ) for both one centrally mounted injector and two outer injectors.

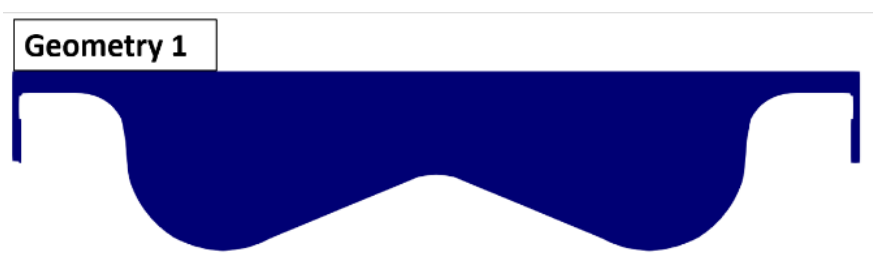

\section{Geometry 2}

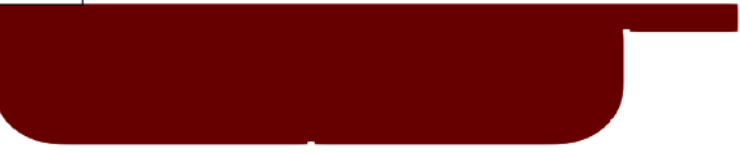

Geometry 3

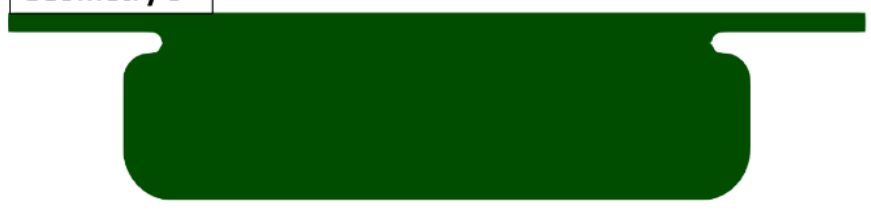

Figure 3, The three different bowl shapes displayed with Geometry 1 (standard), Geometry 2 (removed pip) and Geometry 3 (pips placed at the rim of the bowl)

Geometry 3 was decided with the knowledge that a flat bowl leads to a higher heat transfer through the cylinder head. So, the added pips were expected to drive the flow away from the head surface.

The two outer injectors are placed just at the rim of the bowl for all geometries (see Figure 4) while spraying towards the cylinder center. This gives a changed fluid motion proven to reduce heat transfer [12]. The umbrella angles used, 145 degrees for the reference case, 150 for the two-injector case using Geometry 1 and 160 degrees for the remaining cases, are based on reaching the minimum amount of heat transfer. 


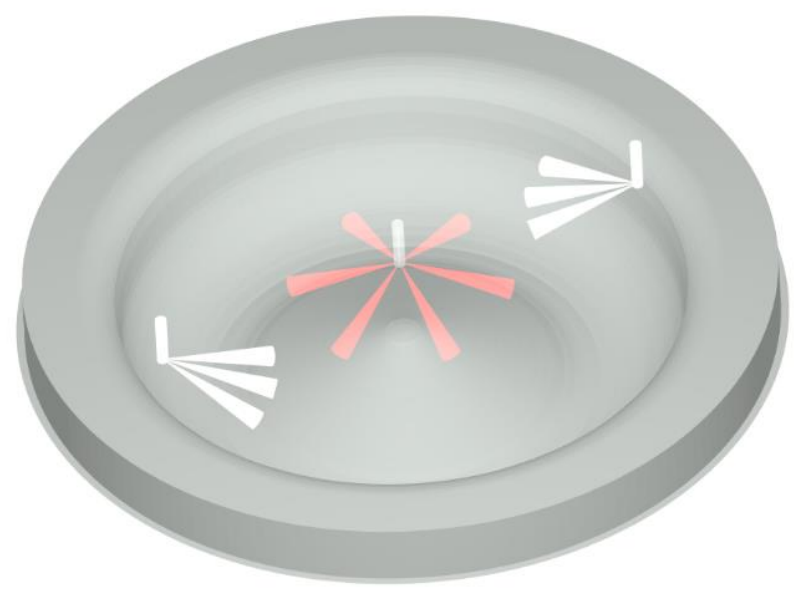

Figure 4, Injector and spray configuration showing the two outer injectors (white) as well as the standard central injector

Compensation has been made for the different bowls to maintain the same compression ratio for all cases. Notable is that the flat bowl will have a smaller surface area compared to the standard case. Since the crevices were found not to impact the heat transfer notably, they were removed for the changed geometry.

The fuel amount per cycle is the same for all cases, $150 \mathrm{mg}$ or $30 \mathrm{bar}$ FuelMEP, with a single injection, split equally between the injectors for the two-injector cases. Same number of holes mean same flow rate for the reference cases and the two-injector cases. Four different injection timings were tested, namely $-7,-4,-1$ and 2 CAD ATDC.

More cases than the ones listed here were tested, but only the most relevant are presented here due to space limitations. All cases were operated at lambda 3.2. In Table 2 the cases are shown. For Geometry 3, only one case SOI is presented here since the results did not show any improvements regarding heat transfer.

Table 2, Design of Experiment (DOE) with a sweep of injection timings and geometries

\begin{tabular}{|l|c|c|c|c|}
\hline Cases & \multicolumn{3}{|c|}{ Injection timings [CAD ATDC] } \\
\hline $\begin{array}{l}\text { Geometry 1 } \\
\text { 1 and 2 injectors }\end{array}$ & -7 & -4 & -1 & 2 \\
\hline $\begin{array}{l}\text { Geometry 2 } \\
\mathbf{2} \text { injectors }\end{array}$ & -7 & -4 & -1 & 2 \\
\hline $\begin{array}{l}\text { Geometry 3 } \\
\text { injectors }\end{array}$ & & & -1 & \\
\hline
\end{tabular}

\section{Results}

This section emphasizes not only how heat transfer through the walls is reduced but also covers the reason for the reduction in heat transfer. The primary outcome is the reduced heat transfer when using multiple injectors with an altered bowl geometry. The definitions of mean effective pressures follow the standard set in a previous study [12].

Page 4 of 9

\section{Reducing Heat Losses}

The structure of the presented results in this section is as follows. Geometries are presented as 1-3 where * indicates that only a centrally mounted injector is used. It follows that Geometry $1 *$ is the reference case. Results are grouped with injection timing for an easy comparison between the different geometries. Notable is that there are four cases for an SOI of -1 CAD ADTC as opposite to the three cases for other injection timings.

For all cases studied here, Geometry 2 with a flat bowl is more favorable regarding heat transfer. Results show that it is mainly a significant reduction in piston heat transfer (see Figure 5). The head heat transfer is a smaller part of the whole meaning that although there is an increase for Geometry 2, there is a total reduction in heat transfer. The heat loss through the liner is similar, and small, for all cases.

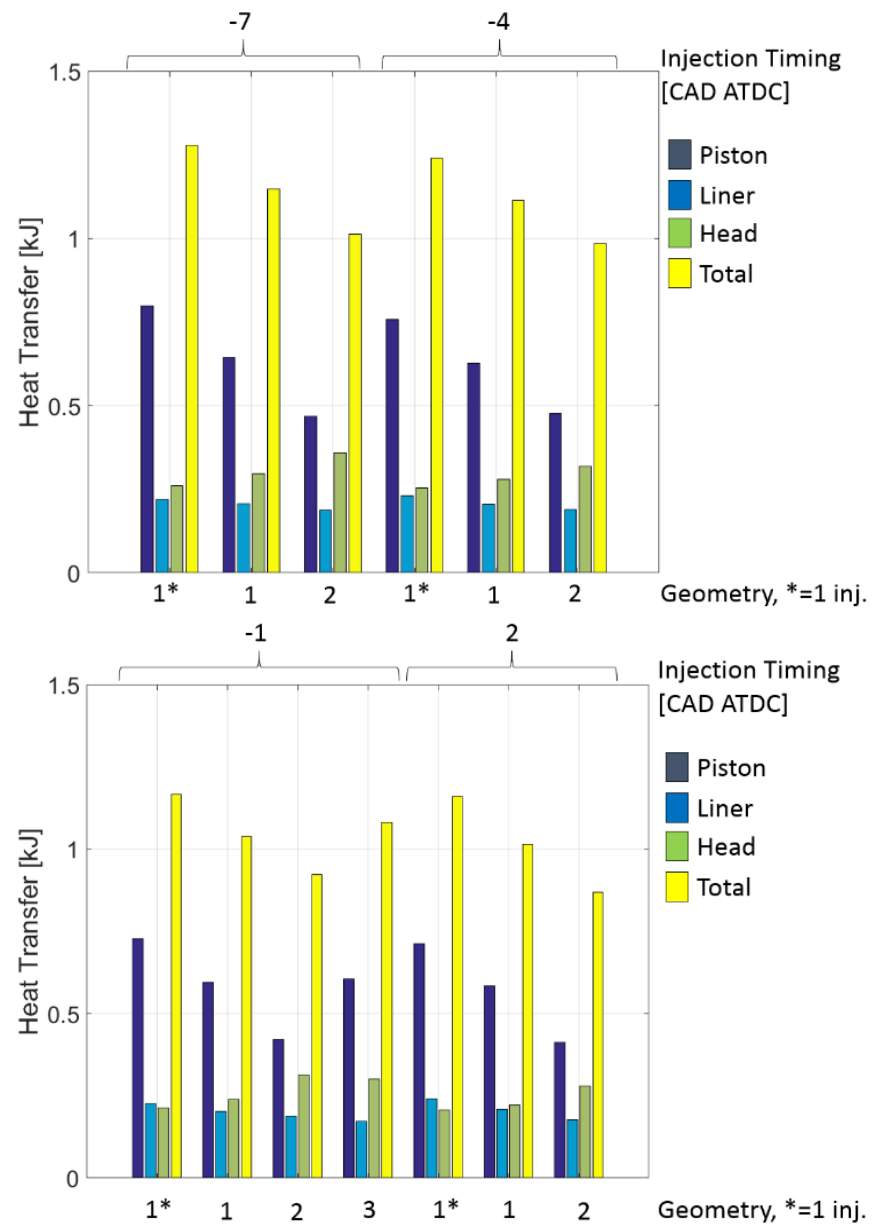

Figure 5, Total heat transfer $[\mathrm{kJ}]$ through the piston, liner, and head for the different cases

The heat transfer reduction is at most $25.1 \%$ or $4.2 \%$ of the fuel energy for the SOI of 2 CAD ATDC compared to the reference case. Compared to the two-injector case using Geometry 1, the reduction is instead $14.3 \%$ or $1.9 \%$ of the fuel energy. As opposed to what was seen in the two-injector case using Geometry 1, for Geometry 2 there is a significant gain in IMEP (see Figure 6). This improvement corresponds to a maximum of $4.5 \%$ or $1.9 \%$ of the fuel energy compared to the reference case, again for the SOI of 2 CAD ATDC. 
Geometry 3 proves to not reduce the heat transfer more than $7.5 \%$ compared to the reference case at -1 CAD ATDC SOI. It is a smaller reduction compared to what the standard geometry would give. However, the IMEP increases $1.7 \%$ compared to the reference case as an opposite to the slight decrease in IMEP when using two injectors with the standard geometry. As opposed to the author's suggestion, Geometry 3 did not indicate a reduction in heat losses through the head.

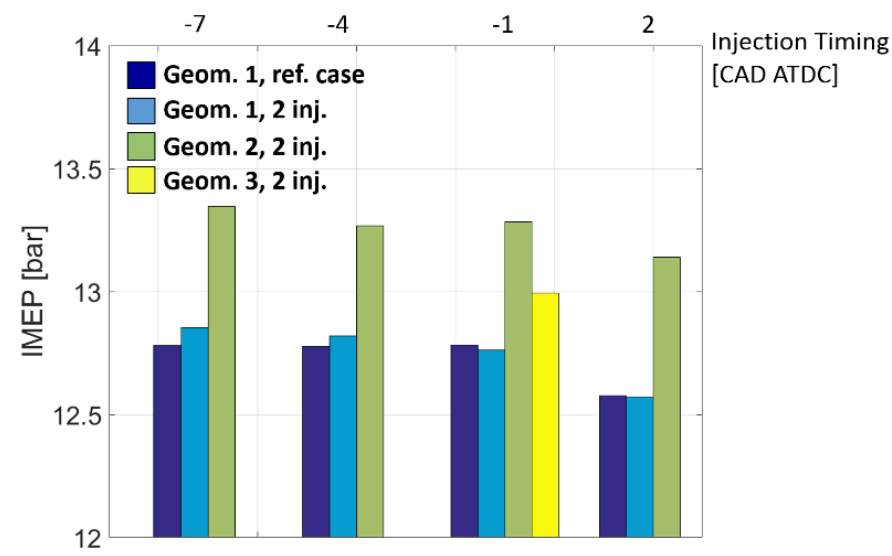

Figure 6, IMEP [bar] for all cases grouped by injection timing

The maximum efficiency in this sweep is found for an SOI of -7 CAD ATDC, and here the gain in IMEP is $4.4 \%$ and the reduction in heat transfer $20.6 \%$ for Geometry 2 compared to the reference case, all with fixed fuel injection. Since all different SOI proved a higher IMEP and lower heat transfer, the concept of using a flat bowl is concluded useful.

The heat transfer is seen to increase more rapidly at an early stage for the reference cases (see Figure 7). This is expected since the hot gases will reach the walls faster with the shorter distance between injector and wall. Comparing the two-injector cases with Geometry 1 and 2, it is seen that the heat transfer is rising earlier for Geometry 1 since the central pip is hit earlier due to the shorter traveling distance for the spray. Without the central pip, as in Geometry 2 and 3, the hot temperature gases will take a longer time to reach the walls.

The time of heat release will have an impact on heat losses.

Consequently, the CA50, CAD at which $50 \%$ of the heat is released, is of importance. Figure 8 shows that the CA50 is relatively constant, with only up to $0.5 \mathrm{CAD}$ perturbation from standard case to twoinjector case. The RoHR and Pressure (see Figure 9) also show only small differences between the cases and are so confirming that the heat release is itself not an explanation for the reduced heat losses.

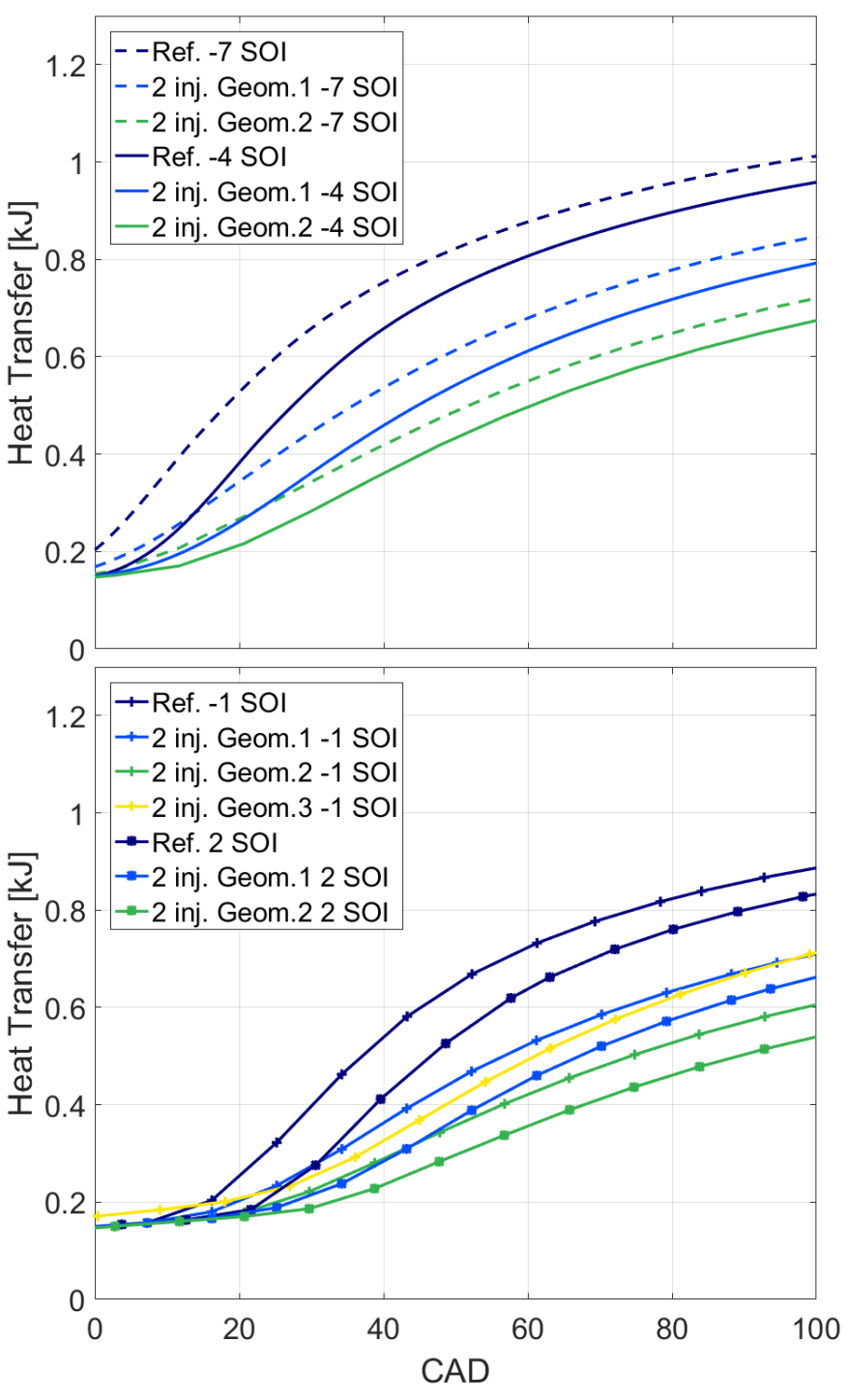

Figure 7, Accumulated heat transfer $[\mathrm{kJ}]$ as a function of crank angle

Better mixing can lead to complete combustion, but in this case, the reference case does not seem to have more complete combustion compared to the two-injector cases, looking at the RoHR (Figure 9). This means that if there are any losses regarding combustion, they are minor compared to the reduction in heat losses achieved by the twoinjector concept. 


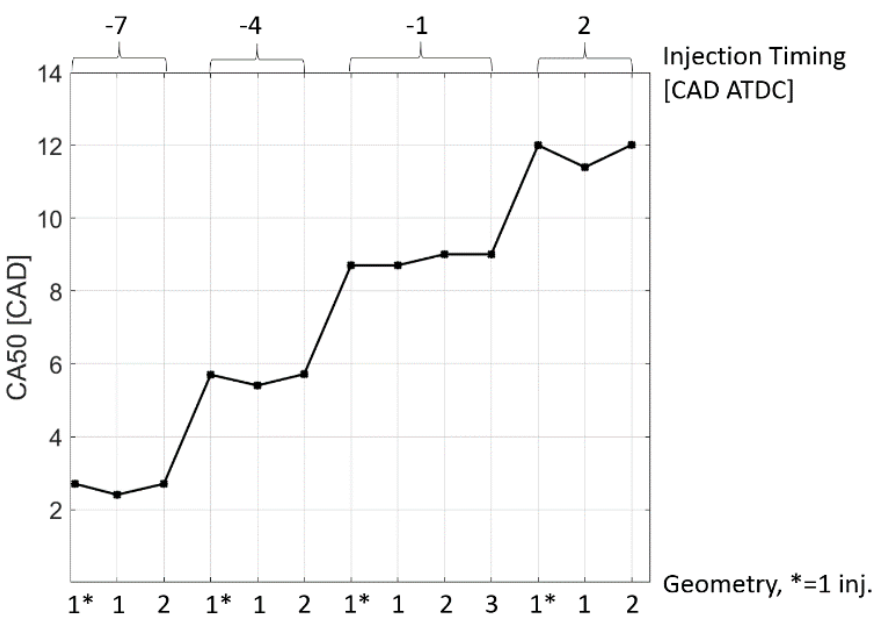

Figure 8, CA50 (CAD at which $50 \%$ of the heat is released) for the different cases
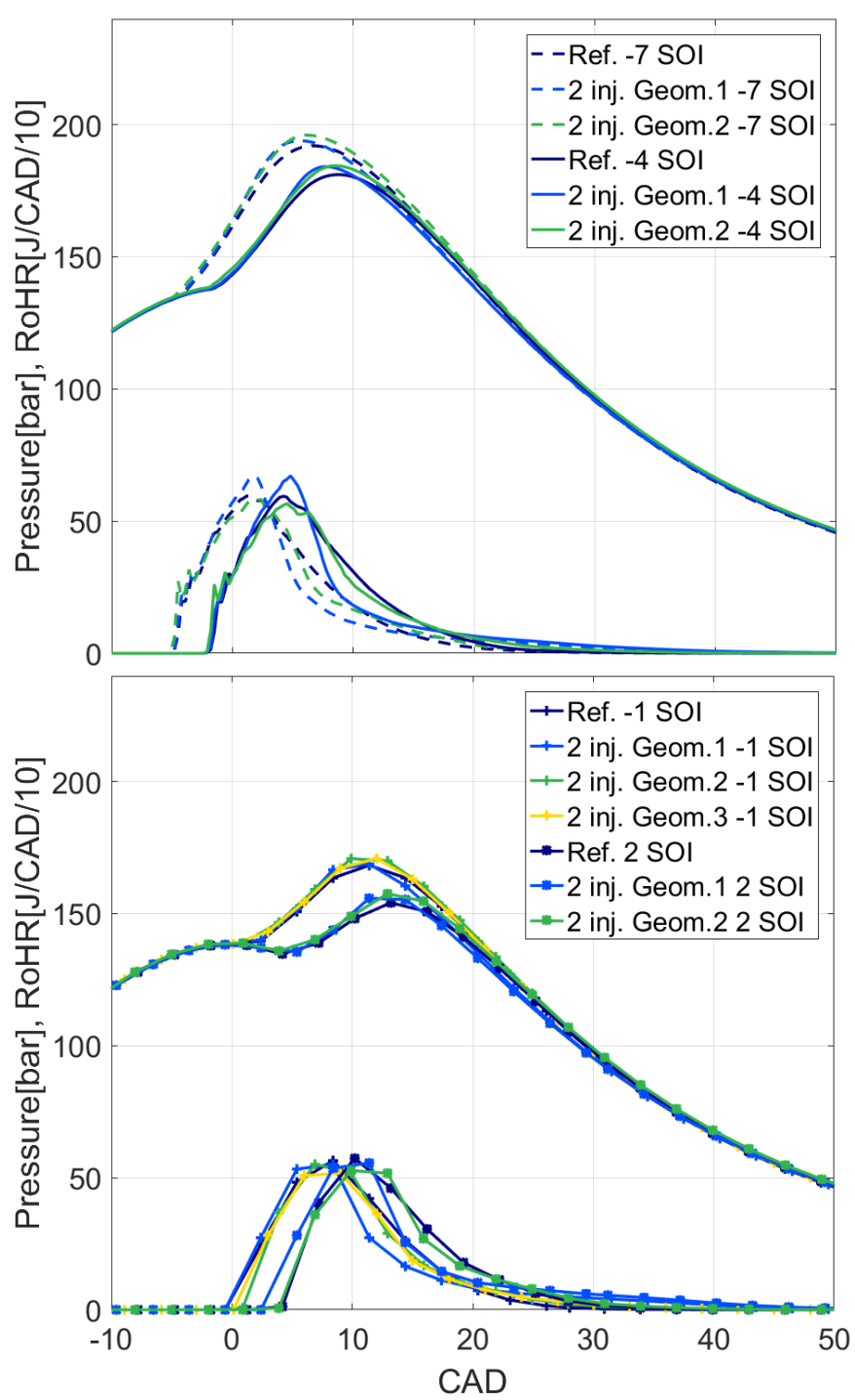

Figure 9, Pressure [bar] and RoHR/10 [J/CAD/10] as function of CAD
Wall area is another driver for heat transfer. Here the wall area (see Figure 10) is changing between the cases even as volume is kept identical. The wall area is smaller for Geometry 2 and 3 where the central pip is removed. This is, therefore, part of the explanation for the lower heat transfer. However, since Geometry 3 has a smaller wall area compared to Geometry 1 but still a higher heat transfer, the wall area is not the full explanation.

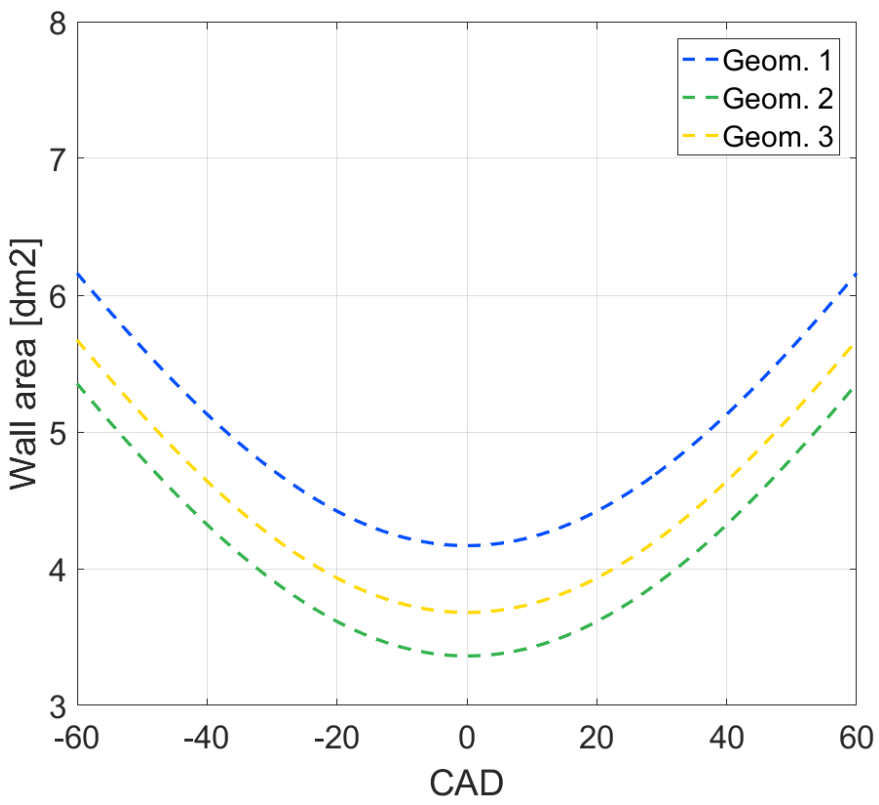

Figure 10, Wall area [dm2] as a function of crank angle

Gas temperature at the cylinder walls is a driver for heat transfer. As investigated earlier, the bulk of the heat losses are through the piston boundary. Figure 11 demonstrates that although the piston temperature is lower for Geometry 2 compared to the reference cases, it is not lower compared to the two-injector cases using Geometry 1. It follows that with a higher average fluid wall temperature, the heat transfer should be higher for Geometry 2. However, as seen before, this is not the case. Notable is that the wall temperature is already higher before combustion for Geometry 2 and 3 compared to Geometry 1 . This means that there is a non-combustion related driver. Such a factor is the turbulence created by the altered flow due to the different bowl geometry.

A measure of the turbulence level at the boundary is the near-wall velocity, as plotted in Figure 12. The velocity is here concluded to be more dependent on the injector configuration than the bowl shape. Geometry 1 and 2 have similar near-wall velocities when using two injectors. For the reference case, the high velocity (around $20 \mathrm{~m} / \mathrm{s}$ higher than the two-injector cases) is created from the spray pattern that is made for proper mixing. It follows that this near wall flow is causing high heat transfer for the reference case. Also, since the nearwall velocity is higher for Geometry 1, comparing two-injector cases of Geometry 1 and 2, it is concluded that this is part of the explanation for the increased heat losses. 


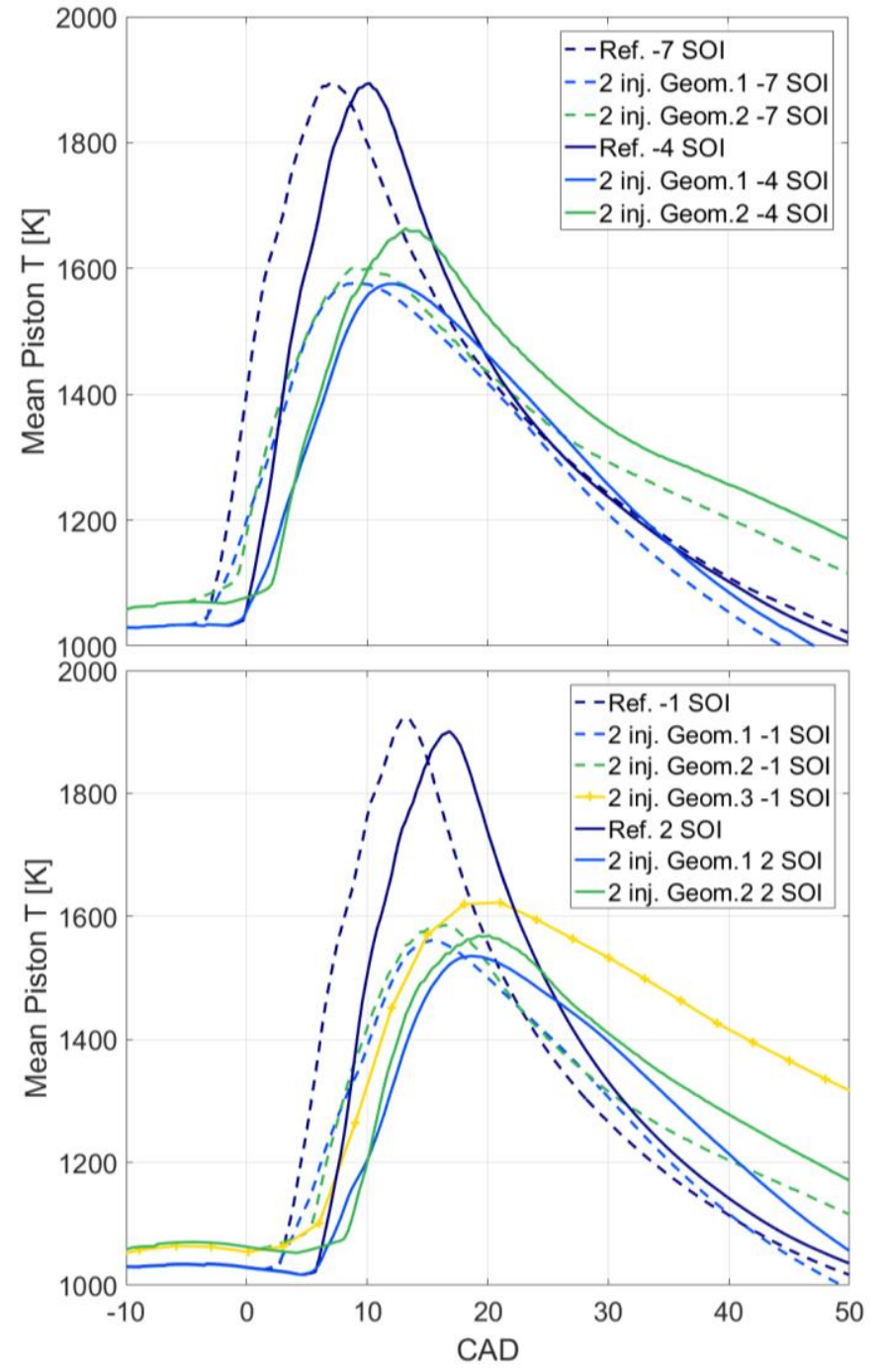

Figure 11, Average fluid wall temperature $[\mathrm{K}]$ as a function of crank angle

The longer tail of wall velocity for Geometry 3 explains the late increase in heat transfer for that case [see Figure 7] as opposed to the other cases. Wall area is large late in the cycle meaning that it is of greater importance to keep the near-wall velocity low at this time. In general, the peak of near-wall velocity coincides with the most rapid rise of heat transfer, showing its importance as a factor for heat transfer.

Wall area and near-wall velocity are together having a more significant impact on the heat transfer compared to the wall temperature for this study. Even if wall temperature is higher for Geometry 2 compared to Geometry 1 , using two injectors, the heat transfer is lower and does not increase as rapidly. This suggests that it is instead the fluid flow, for the two-injector cases, causing the reduced heat transfer and not the high-temperature zones.
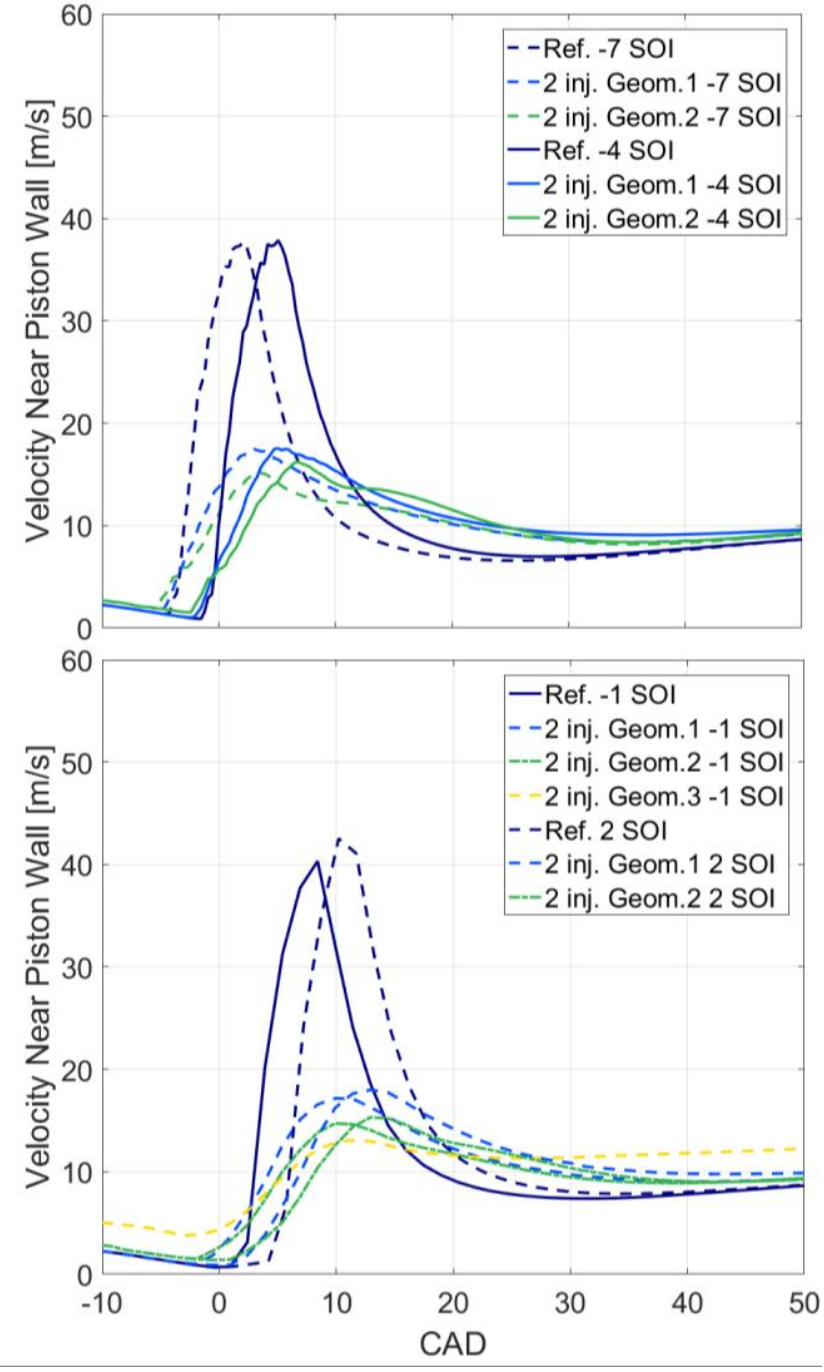

Figure 12, Near wall velocity $[\mathrm{m} / \mathrm{s}]$ for piston as a function of crank angle

As discussed early in this paper, the exhaust energy is not to be treated as a loss for the DCEE concept. Instead, it should be interpreted as a necessary quantity for the second expansion in the LP cylinder. Significant gains in exhaust energy were found for the twoinjector cases compared to the reference case. This is due to the reduced heat transfer. The most significant gain in exhaust energy compared to the reference case was found for an injection timing of 2 CAD ATDC. The percentage gain is $5.6 \%$ for Geometry 2 and $5.0 \%$ for Geometry 1 when two injectors are used compared to the reference case. This corresponds, respectively, to $2.3 \%$ and $2.1 \%$ of the fuel energy. It follows that also for the exhaust energy, Geometry 2 is the best option when using two injectors for all SOI but -1 CAD ATDC.

This extra exhaust energy can potentially be used in a concept such as the DCEE to deliver further work resulting in an improvement of efficiency up to $2.3 \%$-points, depending on the LP-cylinder efficiency, only by the increased exhaust energy. Assuming an LPcylinder efficiency of $30 \%$ gives an efficiency gain of $0.7 \%$-points

Page 7 of 9 


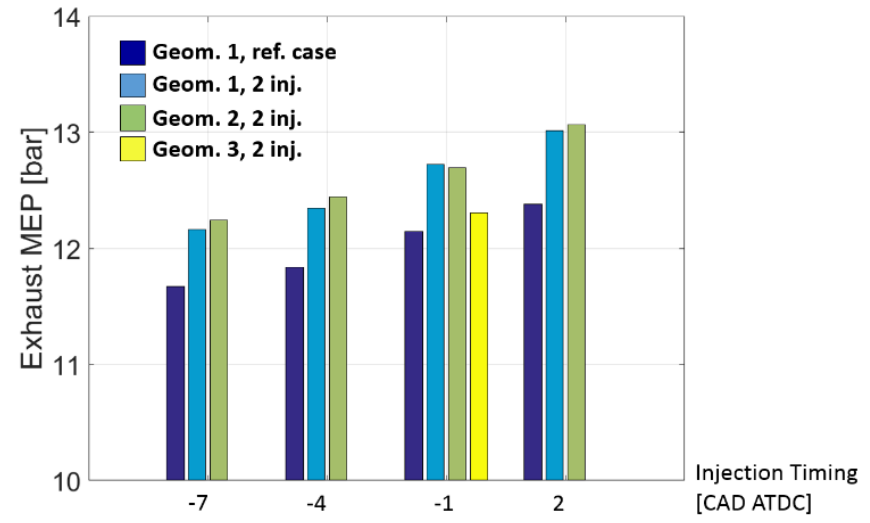

Figure 13, Leftover exhaust energy in the form of Exhaust MEP [bar]

The results of this CFD study showed that two injectors placed at the rim of the bowl could increase indicated efficiency directly if a flat bowl is used. In a concept using exhaust gas re-use, such as the DCEE concept, the efficiency gain can potentially be $4.2 \%$-points depending on how well the exhaust energy can be used.

The driver for this efficiency gain is seemingly a combination of two factors: wall area and fluid velocity at the vicinity of the wall. Nearwall temperature is not one of these factors since it was higher for the flat-bowl cases although they showed lower heat losses.

For future studies, it is interesting to see further how combustion is affected by the changed bowl shape. Emissions such as NOx and soot would be of interest to evaluate but are out of the scope of this study.

\section{Summary/Conclusions}

CFD simulations were conducted to evaluate the level of convective heat transfer reduction by using two injectors in CI engines. While the study was motivated by the DCEE engine application, the two injector concept applies to general CI engine applications, especially when using a flat bowl since this increases the efficiency further. In summary, it was concluded that:

- $\quad$ Reduced heat losses of $25.1 \%$ or $4.2 \%$ of the fuel energy can be achieved by using multiple injectors placed at the rim of a flat bowl

- Increased indicated efficiency of $4.5 \%$ or $1.9 \%$ of the fuel energy achieved for the two-injector case using a flat bowl, showing its benefits compared to the standard bowl

- Increased exhaust energy of $5.6 \%$ or $2.3 \%$ of the fuel energy was achieved and can be used in an exhaust regeneration system such as the DCEE concept.

- Using bumps at the rim of the bowl to reduce head heat transfer gave little or no effect

- A total efficiency increase of $2.6 \%$ from increased exhaust energy and IMEP is possible using two injectors with a flat bowl in the DCEE concept

\section{References}

1. European Commission, "Reducing $\mathrm{CO} 2$ emissions from heavyduty vehicles",

https://ec.europa.eu/clima/policies/transport/vehicles/heavy_en (accessed 2018-05-10)

2. European Environment Agency, "Carbon dioxide emissions from Europe's heavy-duty vehicles"

https://www.eea.europa.eu/themes/transport/heavy-dutyvehicles/carbon-dioxide-emissions-europe (accessed 2018-0923)

3. Caton JA. Comparisons of Global Heat Transfer Correlations for Conventional and High Efficiency Reciprocating Engines. ASME. Internal Combustion Engine Division Fall Technical Conference, ASME 2011 Internal Combustion Engine Division Fall Technical Conference ():327-337. doi:10.1115/ICEF201160017.

4. Noehre, C., Andersson, M., Johansson, B., and Hultqvist, A., "Characterization of Partially Premixed Combustion," SAE Technical Paper 2006-01-3412, 2006, https://doi.org/10.4271/2006-01-3412.

5. Sjöberg, M. and Dec, J., "Combined Effects of Fuel-Type and Engine Speed on Intake Temperature Requirements and Completeness of Bulk-Gas Reactions for HCCI Combustion," SAE Technical Paper 2003-01-3173, 2003, https://doi.org/10.4271/2003-01-3173.

6. Manente, V., Johansson, B., Tunestal, P., and Cannella, W., "Effects of Different Type of Gasoline Fuels on Heavy Duty Partially Premixed Combustion," SAE Int. J. Engines 2(2):7188, 2010, doi: 10.4271/2009-01-2668.

7. Bhavani Shankar, V., Lam, N., Andersson, A., and Johansson, B., "Optimum Heat Release Rates for a Double Compression Expansion (DCEE) Engine," SAE Technical Paper 2017-010636, 2017, doi:10.4271/2017-01-0636.

8. Lam, N., Tuner, M., Tunestal, P., Andersson, A. et al., "Double Compression Expansion Engine Concepts: A Path to High Efficiency," SAE Int. J. Engines 8(4):2015, doi:10.4271/201501-1260.

9. Lam, N., Andersson, A., and Tunestal, P., "Double Compression Expansion Engine Concepts: Efficiency Analysis over a Load Range”, SAE Technical Paper 2018-01-0886, 2018, doi:10.4271/2018-01-0886.

10. Bhavani Shankar, V.S., Johansson, B., and Andersson, A., "Double Compression Expansion Engine: A Parametric Study on a High-Efficiency Engine Concept," SAE Technical Paper 2018-01-0890, 2018, doi:10.4271/2018-01-0890.

11. Okamoto, T. and Uchida, N., "New Concept for Overcoming the Trade-Off between Thermal Efficiency, Each Loss and Exhaust Emissions in a Heavy Duty Diesel Engine," SAE Int. J. Engines 9(2):2016, doi:10.4271/2016-01-0729.

12. Nyrenstedt. G, Al Turkestani. T, Im. H and Johansson.B, "CFD Study of Heat Transfer Reduction Using Multiple Injectors in a DCEE concept" SAE Technical Paper 2019-01-0070, 2019, doi: 10.4271/2019-01-0070

13. Reitz, R., and Diwakar, R., "Structure of High-Pressure Fuel Sprays," SAE Technical Paper $\underline{870598}$, 1987, doi: $10.4271 / 870598$.

14. Amsden, A. A., O'rourke, P. J., and Butler, T. D., KIVA-II: A computer program for chemically reactive flows with sprays (No. LA-11560-MS). Los Alamos National Lab., NM (USA), 1989.

15. Richards, K. J., Senecal, P. K., and Pomraning, E., "CONVERGE (Version 1.4.1) Manual," Convergent Science, Inc., Middleton, WI, 2012.

Page 8 of 9 
16. Sivasankaralingam, V., Raman, V., Mubarak Ali, M., Alfazazi, A. et al., "Experimental and Numerical Investigation of Ethanol/Diethyl Ether Mixtures in a CI Engine," SAE Technical Paper 2016-01-2180, 2016, doi:10.4271/2016-01-2180.

17. Amsden, A. A., "KIVA-3V: A Block Structured KIVA Program for Engines with Vertical or Canted Valves," Los Alamos National Laboratory Report No. LA-13313-MS, 1997.

18. Babajimopoulos, A., Assanis, D. N., Flowers, D. L., Aceves, S. M., et. al., "A fully coupled computational fluid dynamics and multi-zone model with detailed chemical kinetics for the simulation of premixed charge compression ignition engines," International journal of engine research, 6:497-512, 2005, doi:10.1243/146808705X30503.

19. Zeuch, Thomas, Gladys Moréac, Syed Sayeed Ahmed, and Fabian Mauss. "A comprehensive skeletal mechanism for the oxidation of n-heptane generated by chemistry-guided reduction." Combustion and Flame 155, no. 4 (2008): 651-674.

20. Aronsson, U., Solaka, H., Lequien, G., Andersson, O. et al., "Analysis of Errors in Heat Release Calculations Due to Distortion of the In-Cylinder Volume Trace from Mechanical Deformation in Optical Diesel Engines," SAE Int. J. Engines5(4):1561-1570, 2012, https://doi.org/10.4271/2012-01$\underline{1604 .}$

\section{Contact Information}

Gustav Nyrenstedt

Ph.D. Student

Clean Combustion Research Center (CCRC)

King Abdullah University of Science and Technology (KAUST)

23955-6900 Thuwal, Saudi Arabia

sven.nyrenstedt@kaust.edu.sa

Phone no - +966 (0)56 0458328

\section{Acknowledgments}

This work was sponsored by King Abdullah University of Science and Technology (KAUST). The simulations in this work were performed with the computing resources at the KAUST Supercomputing Laboratories. The authors would like to thank Dr. Georgios Markomanolis, previously at KAUST Supercomputing Laboratory, for helpful guidance in post-processing, and Mr. Nhut Lam at Lund University for providing the experimental data for model validation.

\section{Abbreviations}

ATDC

BDC

CA50

CAD

$\mathrm{CO}_{2}$

Cylinder Wall

DCEE

EXMEP

HCCI

IMEPg

IMEPn

$\mathbf{N}_{2}$

$\mathbf{O}_{2}$

PPC

RANS

RCCI

RoHR

SOI

TDC
After top dead center

Bottom dead center

Crank angle degree at which $50 \%$ of the heat is released

Crank angle degrees

Carbon dioxide

Liner, piston and head boundaries

Double compression expansion engine

Exhaust mean effective pressure

Homogenous charge compression ignition

Gross indicated mean effective pressure

Net indicated mean effective pressure

Nitrogen

Oxygen

Partially premixed combustion

Reynolds averaged Navier-Stokes

Reactivity controlled compression ignition

Rate of heat release

Start of injection

Top dead center 